\title{
Negative ion beam characterisation in BATMAN by mini-STRIKE: Improved design and
}

new measurements

G. Serianni', F. Bonomo, M. Brombin, V. Cervaro, G. Chitarin, S. Cristofaro, R. Delogu, M. De Muri, D. Fasolo, N. Fonnesu, L. Franchin, P. Franzen, R. Ghiraldelli, F. Molon, A. Muraro, R. Pasqualotto, B. Ruf, L. Schiesko, M. Tollin, and P. Veltri

Citation: AIP Conference Proceedings 1655, 060007 (2015); doi: 10.1063/1.4916476

View online: http://dx.doi.org/10.1063/1.4916476

View Table of Contents: http://aip.scitation.org/toc/apc/1655/1

Published by the American Institute of Physics 


\title{
Negative Ion Beam Characterisation in BATMAN by mini- STRIKE: Improved Design and New Measurements
}

\author{
G. Serianni ${ }^{1, \mathrm{a})}$, F. Bonomo ${ }^{1,2}$, M. Brombin ${ }^{1}$, V. Cervaro ${ }^{1}$, G. Chitarin ${ }^{1}$, \\ S. Cristofaro ${ }^{3}$, R. Delogu ${ }^{1}$, M. De Muri, ${ }^{1,4}$, D. Fasolo ${ }^{1}$, N. Fonnesu ${ }^{1}$, \\ L. Franchin ${ }^{1}$, P. Franzen ${ }^{5}$, R. Ghiraldelli ${ }^{1}$, F. Molon ${ }^{1}$, A. Muraro ${ }^{6}$, \\ R. Pasqualotto ${ }^{1}$, B. Ruf ${ }^{5}$, L. Schiesko ${ }^{5}$, M. Tollin ${ }^{1}$ and P. Veltri ${ }^{1}$ \\ ${ }^{1}$ Consorzio RFX (CNR, ENEA, INFN, Università di Padova, Acciaierie Venete SpA) \\ ${ }^{2}$ Istituto Gas Ionizzati del CNR, Corso Stati Uniti, 4, I-35127, Padova, Italy \\ ${ }^{3}$ Università degli Studi di Padova, Via 8 Febbraio 2, I-35122 Padova, Italy \\ ${ }^{4}$ INFN-LNL, v.le dell'Università 2, I-35020, Legnaro (PD) Italy \\ ${ }^{5}$ Max-Planck-Institut für Plasmaphysik, D-85748 Garching bei München, Germany \\ ${ }^{6}$ Istituto di Fisica del Plasma (IFP-CNR) - Via Cozzi 53, 20125, Milano, Italy \\ ${ }^{a}$ Corresponding author: gianluigi.serianni@igi.cnr.it
}

\begin{abstract}
The ITER project requires additional heating provided by two injectors of neutral beams resulting from the neutralisation of accelerated negative ions. To study and optimise negative ion production, the SPIDER test facility (particle energy $100 \mathrm{keV}$; beam current 50A) is under construction in Padova, with the aim of testing beam characteristics and to verify the source proper operation. The SPIDER beam will be characterised by the instrumented calorimeter STRIKE, whose main components are one-directional carbon fibre carbon composite tiles. Some prototype tiles have been employed in 2012 as a small-scale version (mini-STRIKE) of the entire system to investigate the features of the beam from BATMAN at IPP-Garching. As the BATMAN beamlets are superposed at the measurement position, about $1 \mathrm{~m}$ from the grounded grid, an actively cooled copper mask is located in front of the tiles; holes in the mask create an artificial beamlet structure. Recently the mini-STRIKE has been updated, taking into account the results obtained in the first campaign. In particular the spatial resolution of the system has been improved by increasing the number of the copper mask holes. Moreover a custom measurement system has been realized for the thermocouple signals and employed in BATMAN in view of its use in SPIDER. The present contribution gives a description of the new design of the system as well as of the thermocouple measurements system and its field test. A new series of measurements has been carried out in BATMAN. The BATMAN beam characterisation in different experimental conditions is presented.
\end{abstract}

\section{INTRODUCTION}

The ITER project requires additional heating provided by two neutral beam injectors (NBI) [1]. To study production and extraction of the beams, resulting from the neutralization of accelerated negative hydrogen-like ions, the SPIDER test facility (particle energy $100 \mathrm{keV}$; extracted beam current $56 \mathrm{~A}$ and $48 \mathrm{~A}$ in hydrogen and deuterium respectively) is under construction in Padua (Italy) [2], with the aim of optimising the source operation and of characterising the beam properties. One of the diagnostics [3] dedicated to the SPIDER beam, constituted by 1280 beamlets arranged in $4 \times 4$ beamlet groups, will be the Short-Time Retractable Instrumented Kalorimeter (STRIKE) [4], whose main components are one-directional carbon-fibre-carbon-composite (CFC) tiles, observed on the rear side by infrared cameras. To verify the capabilities of the diagnostic, prototype tiles have been employed in 2012 in the BATMAN [5] device at IPP-Garching bei München (Germany), as a small-scale version (dubbed mini-STRIKE) of the entire system [6]. As the optics of the BATMAN beam is such that the divergence is larger than $1^{\circ}$, at the measurement position the beamlets are largely overlapping; Hence, a copper mask was placed in front of the CFC

Fourth International Symposium on Negative Ions, Beams and Sources (NIBS 2014)

AIP Conf. Proc. 1655, 060007-1-060007-8; doi: 10.1063/1.4916476

2015 AIP Publishing LLC 978-0-7354-1297-2/\$30.00

060007-1 
tiles, provided with some holes creating a structure similar to the expected two-dimensional pattern of the energy flux associated to the SPIDER beam. It was found that mini-STRIKE can resolve the beam features on the beamlet scale. This result was confirmed by a series of measurements carried out in the test stand of negative NBIs at National Institute of Fusion Science, Toki (Japan) [7]. Altogether these results pointed towards the possibility of improving the spatial resolution of the system employed in BATMAN. The present paper gives a description of the new design of the mini-STRIKE for BATMAN together with the thermocouple measurement system realised to monitor the temperature of the components exposed to the beam and to calorimetrically measure the power associated to the beam. The data analysis procedure will be described and finally some examples of experimental findings will be given.

\section{DESIGN AND INSTALLATION OF THE NEW SYSTEM}

As already mentioned, the first version of the water cooled copper mask was machined with a pattern comparable to that of the SPIDER grids: 8 holes were drilled with $10 \mathrm{~mm}$ diameter and $30 \mathrm{~mm}$ pitch, as depicted in FIGURE 1. The experimental campaign performed in 2012 [8] confirmed the suitability of the diagnostic to resolve the beam properties on the spatial scale of the beamlets. However, the spacing and number of holes in the copper mask were not enough to provide a thorough characterisation of the beam features. So in preparation of another experimental campaign the copper mask was modified with the primary scope of increasing the spatial resolution for the characterisation of BATMAN beam.
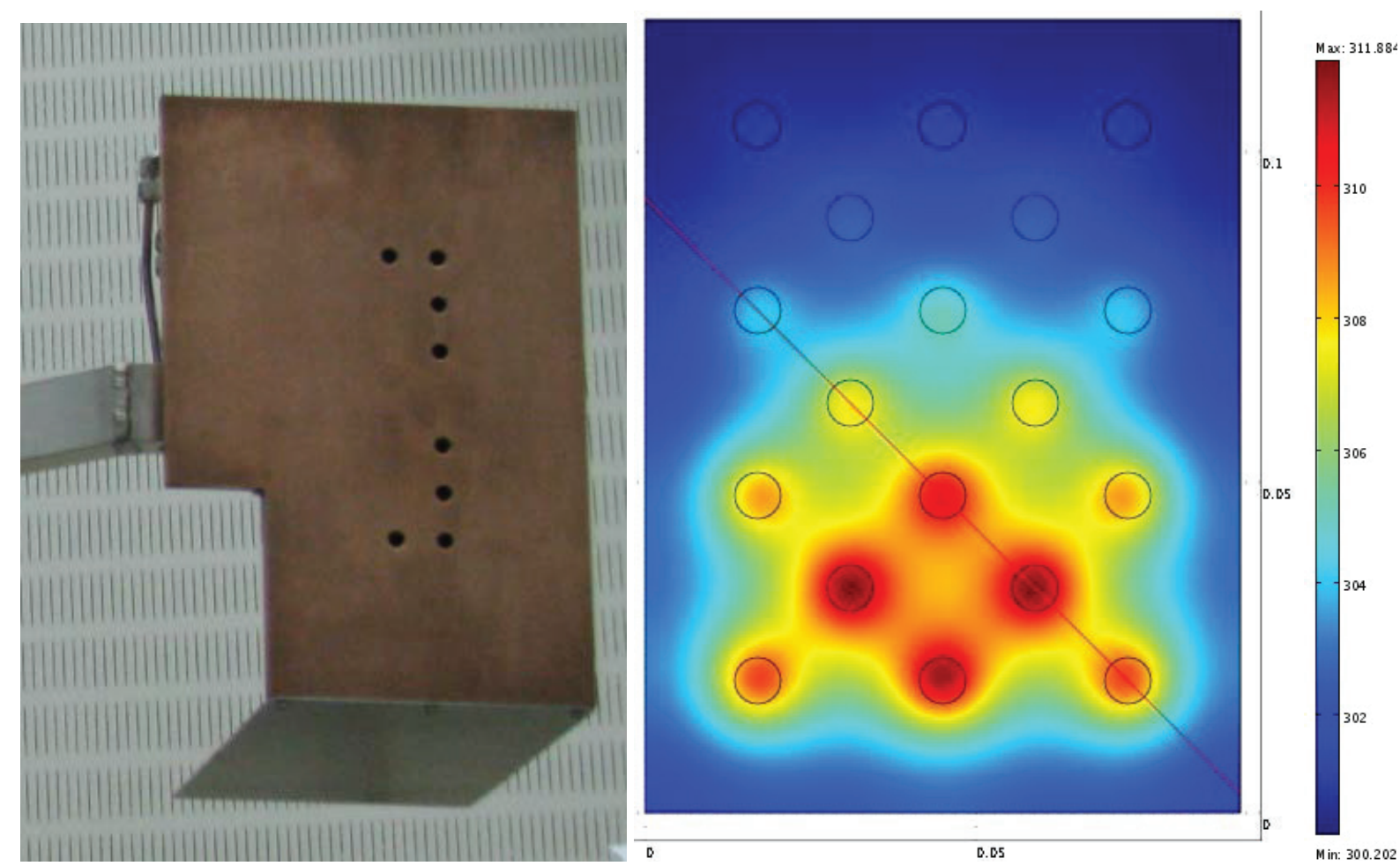

FIGURE 1. Photo of copper mask used in 2012 BATMAN campaign [6] (left). Temperature profile expected on the rear side of one of the CFC tiles (right) with the new copper mask design; the circles indicate the position of the holes in the copper mask

With respect to the earlier version of mini-STRIKE, in the present design the beamlet-like structures corresponding to the holes in the copper mask are slightly smaller $(7 \mathrm{~mm}$ diameter) and closer, in a honeycomb arrangement with $14 \mathrm{~mm}$ between adjacent rows or columns (FIGURE 1). The new design is a compromise between fine sampling of the beam and clarity of the temperature profile to be detected, considering that, with closer holes, a larger overlap of the neighbouring peaks is expected. In such conditions solely 2D studies can constitute the analysis procedure unlike the previous case, when up to a certain time the beamlet-like structures evolved essentially independently. The new mask design corresponds to drilling a larger number of holes in the copper mask; the 
corresponding expected temperature profile on one of the tiles is shown in FIGURE 1. A photo of the new system with and without the CFC tiles is shown in FIGURE 2: it can be seen that the earlier apertures have been closed by suitable threaded caps; moreover, the thermocouple located on the mask as well as those sitting on the back of the tiles are displayed. FIGURE 2 shows also the complete system inside the BATMAN vessel.
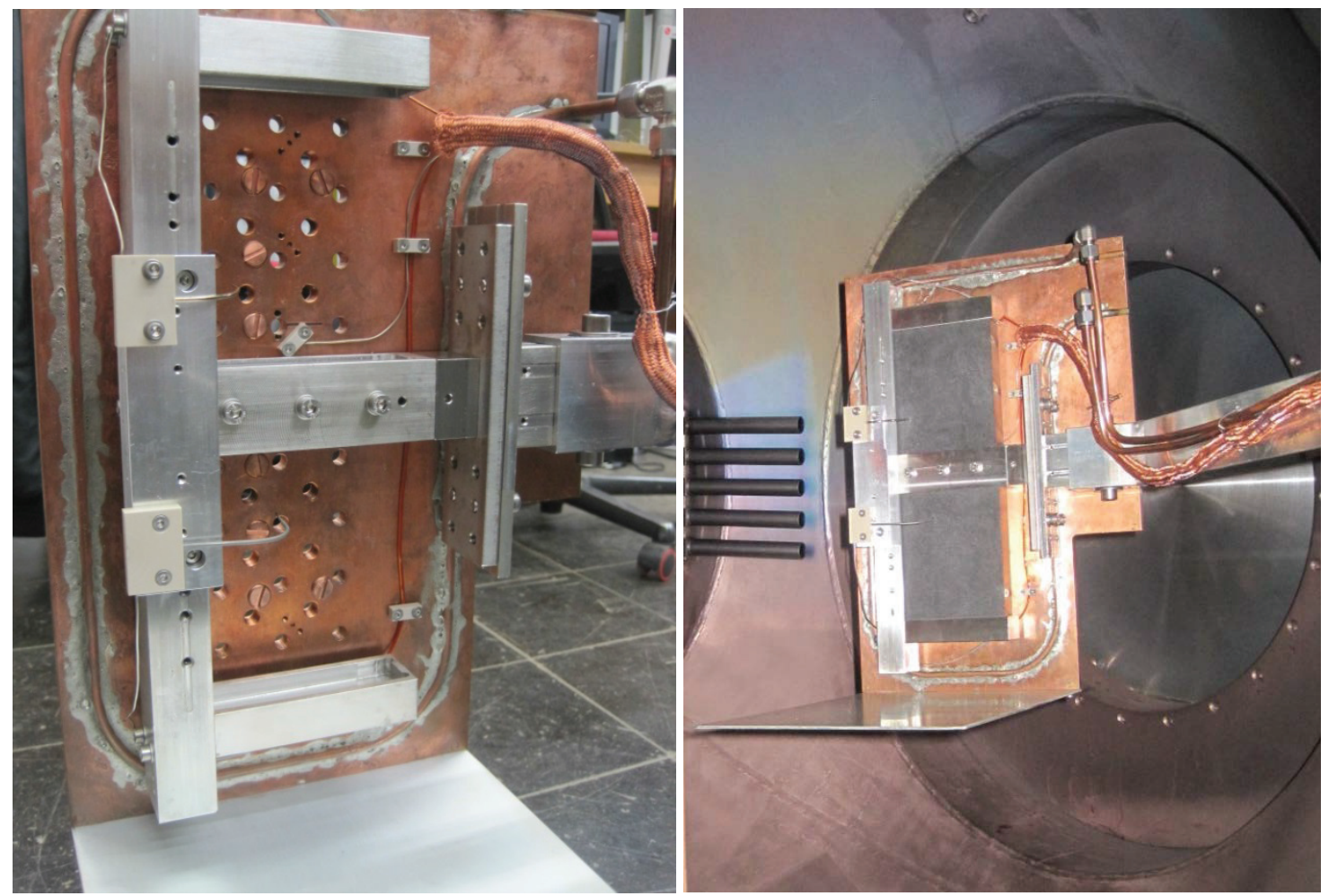

FIGURE 2. New design of mini-STRIKE in BATMAN: new mask (left); complete system inside BATMAN vessel (right); the flange towards the beam source can be seen on the right-hand side

\section{THERMOCOUPLE CONDITIONING AND DATA ACQUISITION SYSTEM}

Mini-STRIKE is provided with several thermocouples for calorimetry and protection of the system [9]. The circuit used for the conditioning and the acquisition of the thermocouple signals in the mini-STRIKE installed in BATMAN during the 2014 experimental campaign is a revised version of the circuit described in [10] which was dedicated to the measurement of thermocouple signals on SPIDER grounded grid while surviving the accelerator breakdowns. With respect to that circuit, the electric insulation requirements are relaxed because the position of mini-STRIKE, namely in the beam line, excludes voltage peaks on the thermocouple signals. Therefore the relays used in the previous version are not needed. This allows a very simple firmware for the data acquisition and very low power consumption. Moreover it allows a faster acquisition rate up to $\mathrm{kHz}$ because no time is lost for the time $O N$ and time $O F F$ of the relay.

The thermocouple cables are connected to the electronic circuit, shown in FIGURE 3, which performs filtering, amplification, acquisition and data transmission. The circuit board is placed inside a metal box acting as EMI shielding for the signals. The inputs for the electronic board are the voltage signals coming from the thermocouples located in vacuum and on the inlet and outlet pipes for mask cooling. Since data transmission is via optical fibre, the data are digitized by one ADC before being sent to the PC for storage. Therefore a microprocessor (Programmable Interface Controller PIC) drives two multiplexers (1 manages 4 channels, MUXs in FIGURE 3 ) which activate the 8 channels in turn. In particular one channel is used for the thermocouple mounted on the copper mask, 2 channels for the sensors on the rear side of the CFC tiles, 2 more channels for the inlet and outlet temperature of the cooling water and one is dedicated to the LM35 sensor (see below). The remaining two are spare channels. Before being multiplexed, the signals from the thermocouples are filtered by an LC filter ( $\mathrm{T}$ configuration) at $500 \mathrm{~Hz}$ in order to eliminate any RF noise. 
The cold junction compensation is performed by a precision LM35 integrated-circuit temperature sensor (Temp. sensor in FIGURE 3) which detects the temperature on the electronic board. This signal is used to compensate the input temperatures. The circuit is powered by an on-board $0-5 \mathrm{~V}$ power supply, whose input from the $220 \mathrm{~V}$ BATMAN electric network is filtered to guarantee a stable voltage to the circuit. Since the amplifier works just with positive voltages, to allow measurements of temperatures lower than room temperature, which results in negative voltages, a $-1.235 \mathrm{~V}$ offset was implemented in the power line. This allows measurements from about $-190^{\circ} \mathrm{C}$ to about $+650^{\circ} \mathrm{C}$ with respect to room temperature.

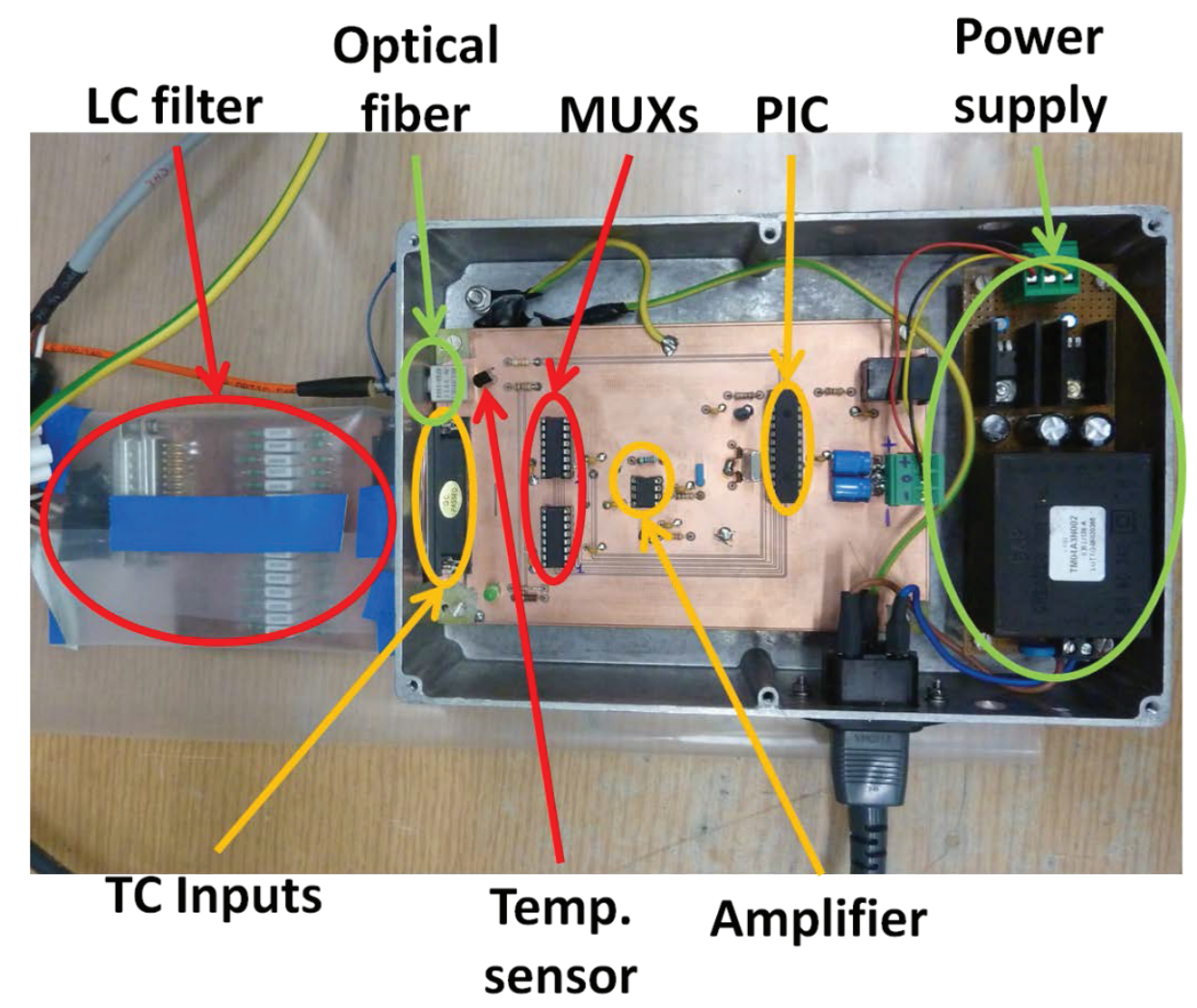

FIGURE 3. Schematic of electronic board for mini-STRIKE thermocouple data conditioning and acquisition.

When the channel is selected by the multiplexer, the signal is amplified (224 times) and digitized by the ADC. An Optical Transmitter sends the data along the fibre. The transmitter unit is based on a Microchip PIC18F2423 microcontroller, chosen according to the following specifications: small package, 12bits ADC resolution, RS232 peripheral, low power consumption (nanoWatt Technology), possibility to program it by C language. The PIC microcontroller is clocked by an external $4 \mathrm{MHz}$ crystal oscillator and its main purpose is to acquire the 8 analogue channels and to transmit them using the internal RS232 peripheral. The acquisition frequency for the 8 available channels is $10 \mathrm{~Hz}$, though it can be much higher; this value was chosen in order to save storage memory.

The acquisition procedure is the same as for the system used in analogous measurements in the test stand of LHD neutral beams [7]. The transmitter starts to read all the analogue channels from 1 to 8 when the circuit is powered. Before starting a new reading cycle, the transmitter sends the data via the RS-232 internal peripheral: data are converted by an electro-optical converter and sent to the receiver unit through an optical fibre. Each acquired temperature has a 12 bit resolution but it is sent as 2 bytes via the RS-232 to simplify the code in the transmitter and in the receiver unit. Each transmission starts with a 2 bytes header to let the receiver unit synchronize with the transmitter and then 2 additional bytes after the header for each channel from the first up to the eighth. Thus a total of 18 bytes ( 2 bytes for the header and 2 bytes per channel) are necessary to have a sample for all the channels at the receiver side. When the transmission is completed, the transmitter restarts its cycle, which is stopped only when the board is powered off. The optical head for the transmission is normally off and it is powered just for sending data when the full packet ( 8 measurements) is ready. The transmission is not continuous but in burst mode; in fact no transmission is made during the sampling section as the transmitter waits for the complete packet to be ready. 
The prototype receiver unit converts the optical signal generated by the transmitter into a RS232 signal in order to interface to the PC for data storage by the MDSPlus software tools [11]. The visualization is performed directly on the acquisition PC. The receiver unit is placed outside the vessel in the experimental room close to the storing PC and it has an external power supply to feed the optical head and the MAX-232 converter.

In the following picture (FIGURE 4), an example of the evolution of the mask temperature during five subsequent plasma pulses (BATMAN was usually operated with $4 \mathrm{~s}$ beam-on and 180 s beam-off time) is depicted. The improvements of the electronic circuit allowed acquisition of temperature signals with an accuracy of $0.2^{\circ} \mathrm{C}$.

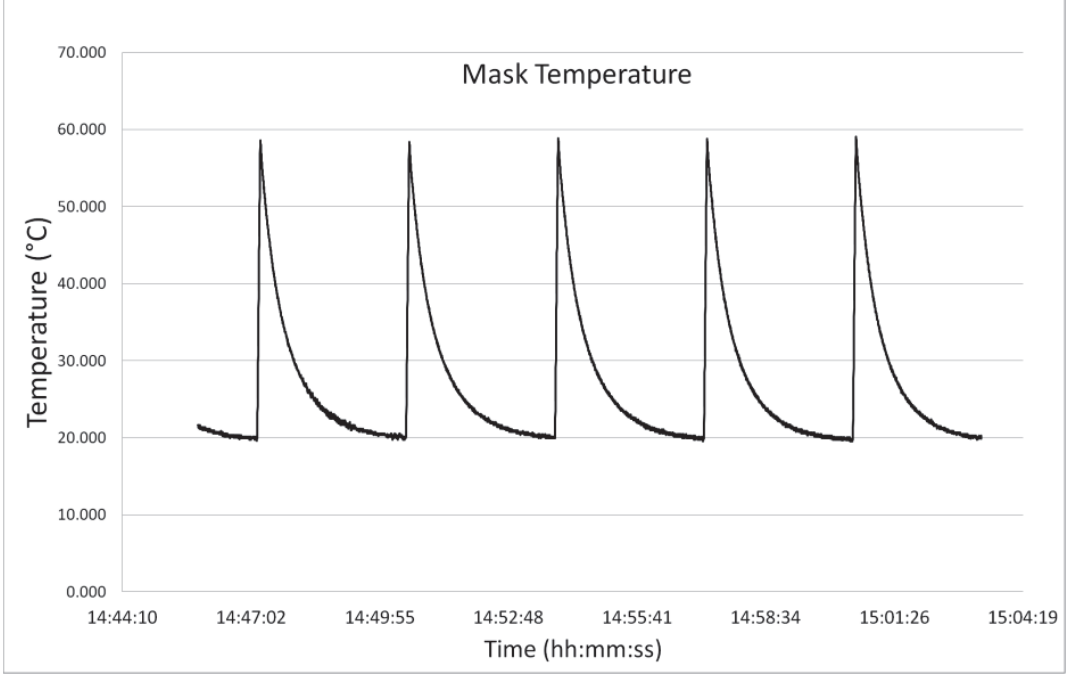

FIGURE 4. Example of temperature detected at the copper mask surface.

\section{DATA COLLECTION AND ANALYSYS OF THERMAL CAMERA DATA}

The mini-STRIKE infrared camera records with a frequency of 25 frames per second for 20 s (for a total of 500 frames per pulse) and each frame consists of a matrix of $640 \times 480$ pixels whose values correspond to the temperature measurements. The thermal camera is already absolutely calibrated, taking into account the measured emissivity equal to 1 of the CFC tile [12] and the object distance $(1 \mathrm{~m})$. The error associated to the measured temperature is $2 \mathrm{~K}$ or $2 \%$ (the greater error between these two values has to be considered). The camera is triggered $3.2 \mathrm{~s}$ before the beam starts (80th frame), in order to measure the background temperature before the beam-on phase. The beam pulse lasts $4 \mathrm{~s}$, hence the beam-off phase starts at frame 181.
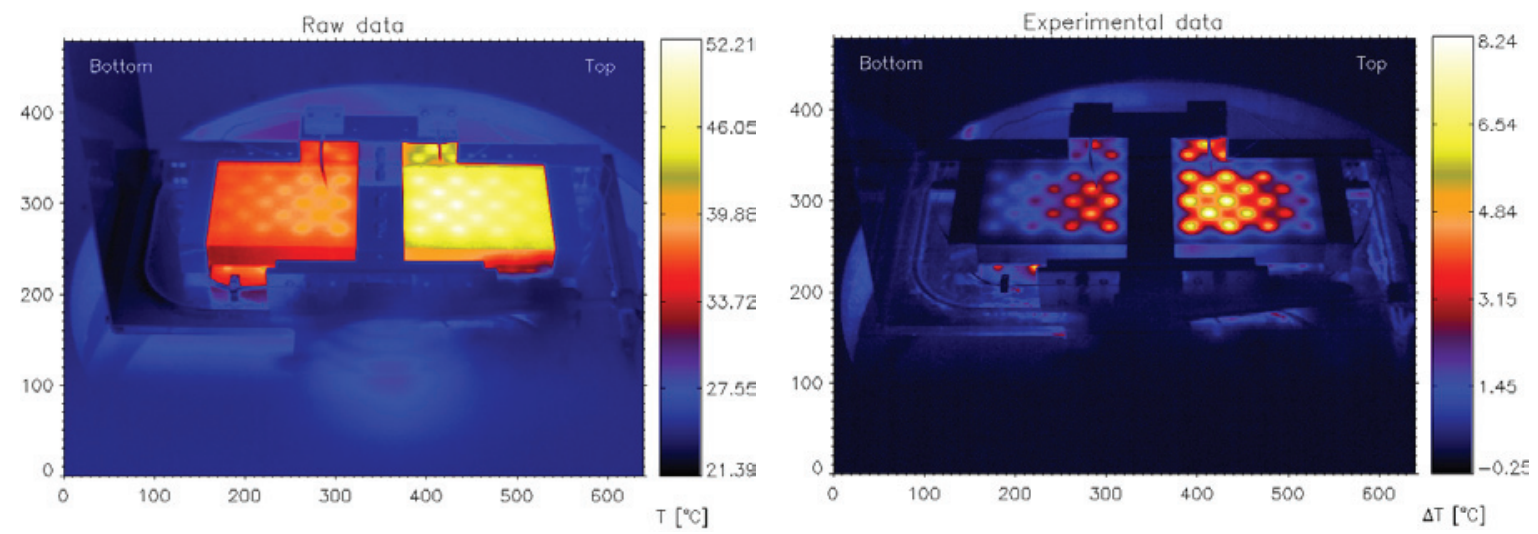

FIGURE 5. Infrared camera data before (left) and after background subtraction (right).

The programming language used for developing the data analysis program was IDL [13]. For each pulse, the programme considers the frames in which the beam is on (frame range: 80-180), after subtracting the background pattern (average over 25 frames, corresponding to an interval of $1 \mathrm{~s}$, before the beam start). Hence only temperature 
increases with respect to the background are considered in the following (FIGURE 5). After a perspective correction (necessary because the viewing angle is $50^{\circ}$, compare FIGURE 5 and FIGURE 6), the region relevant for further analyses is selected, i.e. the region corresponding to the two tiles, excluding the shadows of the two thermocouples and the zone in between the two tiles. Furthermore, the dimension of the $\mathrm{x}$-axis and $\mathrm{y}$-axis are scaled from pixels to millimetres.
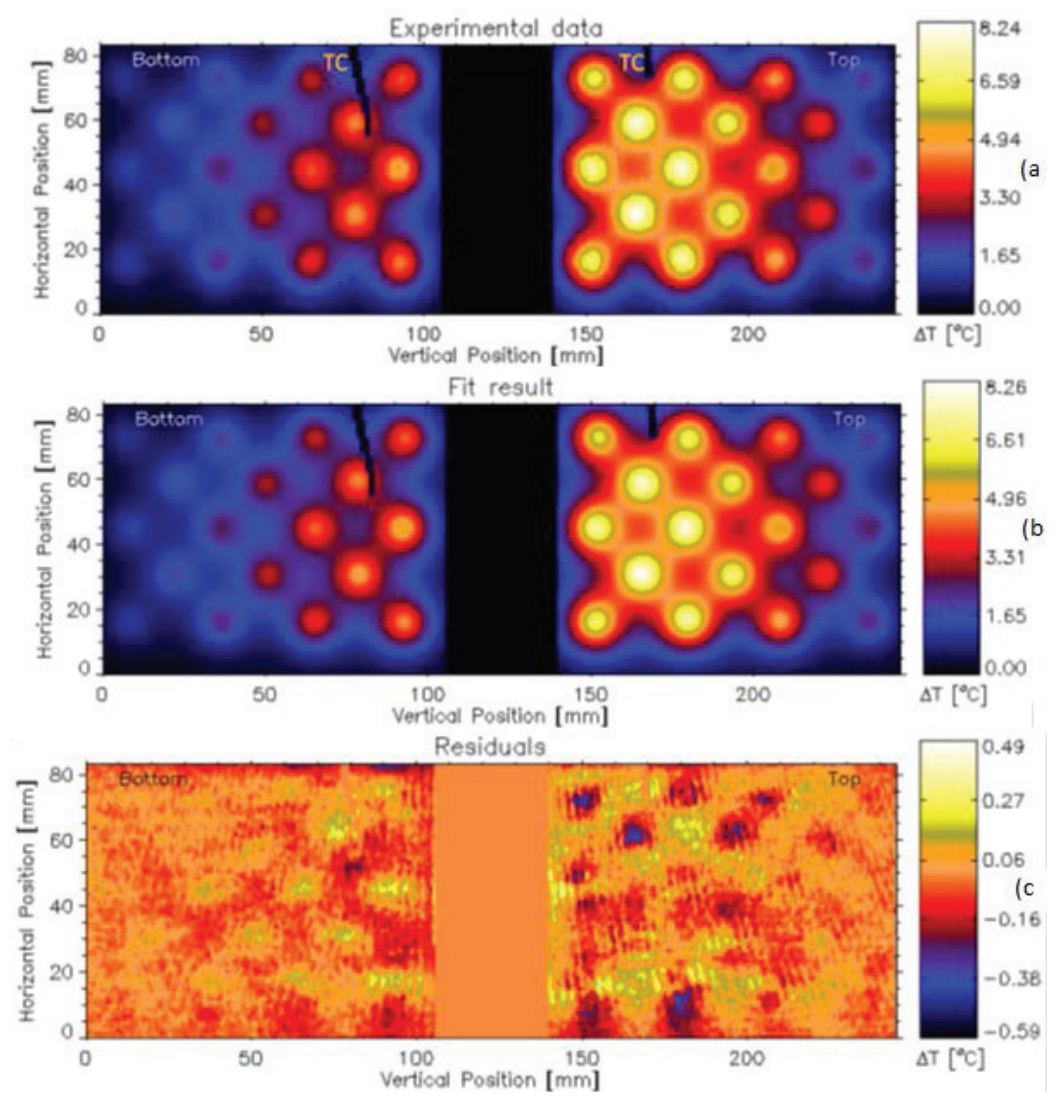

FIGURE 6 Two-dimensional pattern of temperature (a); result of fitting (b); corresponding residuals (c).

The fitting procedure involves three steps with the ultimate aim of obtaining a description of the beam profile. At first a two-dimensional fit with a Gaussian function is applied to small areas surrounding the expected position of each peak in order to get a precise determination of the position of the centres of the beamlet-like structures; this procedure is performed at frame 105 (corresponding to $1 \mathrm{~s}$ after the pulse beginning), when the overlap of the tails of the different structures is not strong yet so that the evaluation of the centre positions is not affected by the neighbouring structures. The peak positions are then treated as constants in the second two-dimensional fit, which consists in fitting the superposition of 36 two-dimensional bell-shaped functions due to all 36 beamlet-like structures; specifically the fitting function has the following form:

$$
\sum_{i=1}^{36} a_{i}\left(\cosh \sqrt{\left(x-x_{c, i}\right)^{2} w^{2}+\left(y-y_{c, i}\right)^{2} w^{2}}\right)^{-q}
$$

which is a replica for each structure of the function that was found to fit best the thermal pattern measured on the rear side of the CFC tiles subjected to a point-like laser beam on the front side (modified two-dimensional Hubbert function [12]). The width of the structure, which is related to the parameters $w$ and $q$, is assumed to be the same for all structures since it is determined by the uniform thermal characteristics of the CFC tiles and by the shape of the holes on the copper mask. The two-dimensional experimental pattern and the corresponding fit results are compared in FIGURE 6; the residuals show that the two-dimensional function fits the data better than $10 \%$. The estimation of 
the error on the fit parameters is performed by inverting the Hessian matrix of the $\chi^{2}$ function; the diagonal elements of the inverse matrix are assumed as the variances of the fit parameters [14].

Out of the parameters determined via the second fit, the amplitudes of the 36 structures are the most important ones: they represent the temperature profile on the back of the tiles due to the beam power deposition, so they correspond to a spatial sampling of the energy flux associated to the beam. As the scope of the present measurements is the assessment of a possible vertical non-homogenity of the beam, an asymmetric function has been adopted to fit the two-dimensional pattern of the peaks; the function is composed by a parabola in the horizontal direction and a skewed Gaussian curve in the vertical direction:

$$
M_{s g} \exp \left(-\frac{\left(y-y_{o}\right)^{2}}{2 \omega^{2}}\right)\left[1+\operatorname{erf}\left(\alpha \frac{y-y_{o}}{\sqrt{2} \omega}\right)\right]\left[\left(\frac{x-x_{o}}{b}\right)^{2}+1\right]
$$

Free parameters in the fitting procedure are: the maximum value of the beam $M_{s g}$, the horizontal and vertical position of the centre of the beam $\left(x_{0}, y_{\mathrm{o}}\right)$, the beam width $\omega$, a skewness parameter $\alpha$ (for $\alpha=0$ the symmetric form of the usual Gaussian is recovered) and the parameter $b$, determining the width of the horizontal parabola.

Perveance scan
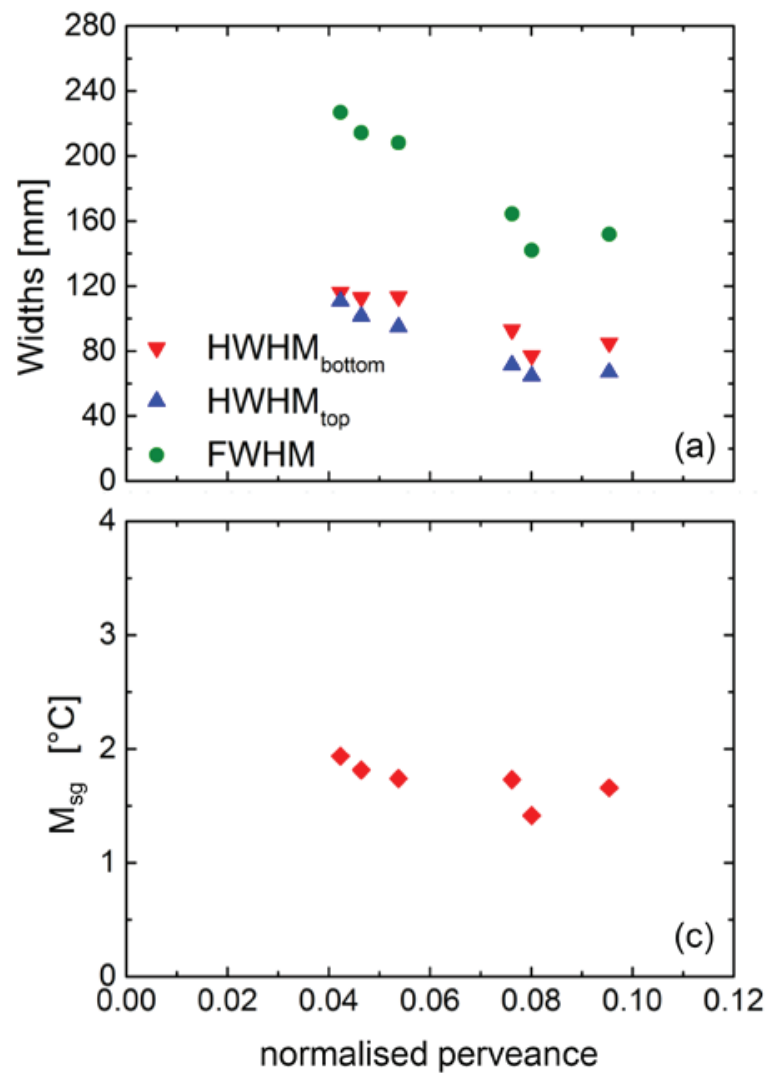

Perveance scan
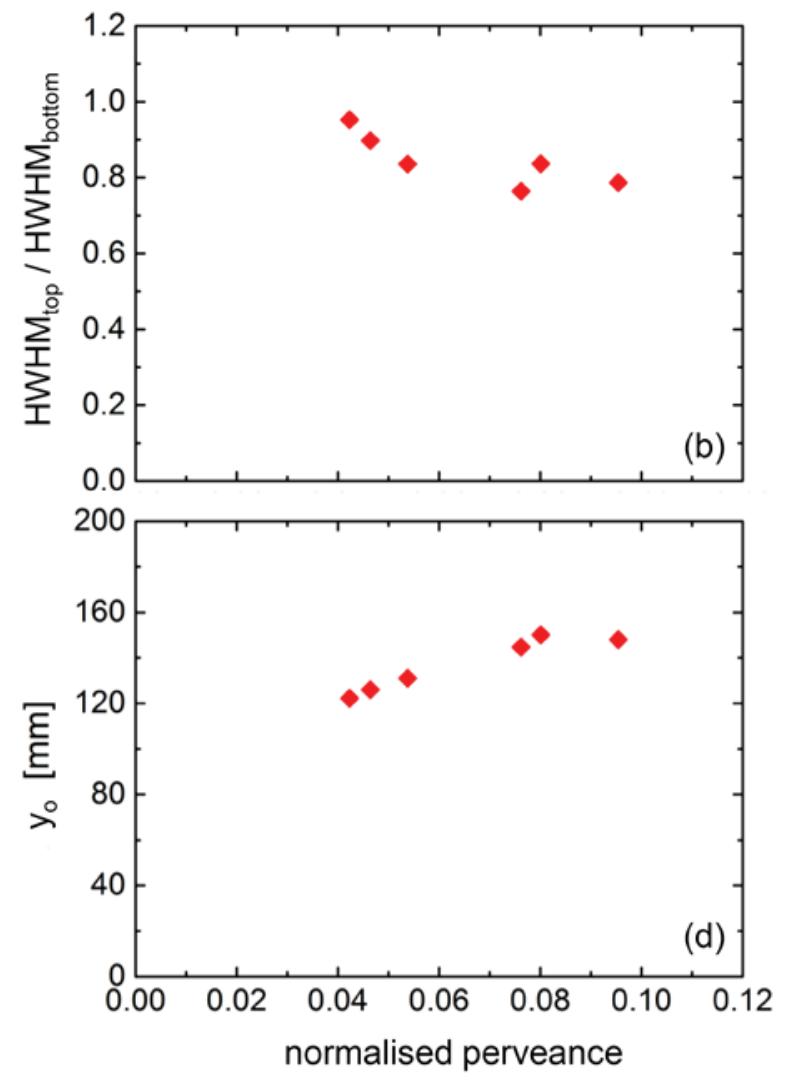

FIGURE 7. Result of two-dimensional fitting as a function of the normalised perveance: HWHMs and FWHM (a); ratio of top HWHM divided by bottom HWHM (b); maximum beam value $M_{s g}$ (c); vertical position of the beam centre (d) [15].

\section{PRELIMINARY RESULTS}

Various experimental campaigns have been performed by changing several source parameters, like RF power, bias current, source pressure, extraction and acceleration voltage and with both plasma drifts (up and down) [15]. For instance, the dependence on perveance of the parameters associated to the two-dimensional fit to the amplitudes 
of the single peaks has been investigated, by performing a scan with drift up and different extraction and acceleration voltages. When considering the widths at half maximum (FIGURE 7), the HWHM bottom $_{\text {and }}$ the $\mathrm{HWHM}_{\text {top }}$ are slightly different, resulting in a top-bottom asymmetry: on the lower tile a greater HWHM is observed. Thus their ratio, plotted in FIGURE 7 is not always equal to one. A similar asymmetry is found by beam emission spectroscopy [16], which was operated simultaneously with mini-STRIKE. The reason of such asymmetry, which depends on the direction of the magnetic filter field (the skewness is consistent with the filter field), is under investigation; it is possible that source conditioning plays a role.

FIGURE 7a shows additionally the FWHM (i.e. the sum of the two HWHMs), which monotonically decreases with increasing normalized perveance. It is worth noting that the FWHM shows a similar trend as the divergence, suggesting a correlation between these two parameters. The maximum temperature increase (which is $M_{s g}$ ) is almost constant within the errors. The vertical position $y_{o}$ of the maximum beam value can be used as a measure for the beam shift. For the present scan, the maximum position moves (it steadily increases) with increasing perveance.

\section{CONCLUSIONS}

The present paper describes the realisation and use of an upgraded version of a diagnostic calorimeter, dubbed mini-STRIKE, to characterise the BATMAN beam. Despite requiring a more careful and lengthy data analysis procedure, the diagnostic system worked and gave new interesting information concerning the detailed twodimensional structure of the beam. The novel information will have to be analysed in detail and interpreted by means of numerical simulations.

\section{ACKNOWLEDGMENTS}

The work leading to this publication has been funded partially by Fusion for Energy under the Contract F4ERFX-PMS_A-WP-2014. This publication reflects the views only of the author, and Fusion for Energy cannot be held responsible for any use which may be made of the information contained therein.

The views and opinions expressed herein do not necessarily reflect those of the ITER Organization.

\section{REFERENCES}

1. ITER Physics Basis Editors, Nucl. Fusion 39, 2495 (1999)

2. P. Sonato et al., Fusion Eng. Des. 84, 269 (2009)

3. R. Pasqualotto et al., Rev. Sci. Instrum. 83, $02 \mathrm{~B} 103$ (2012)

4. A. Rizzolo et al., Fusion Eng. Des. 85, 2268 (2010)

5. E. Speth et al.,Nucl. Fusion 46, S220 (2006); U. Fantz et al., Nucl. Fusion 49, 125007 (2009).

6. M. De Muri et al., Fusion Eng. Des. 88, 1758-1763 (2013)

7. V. Antoni et al., Design, Installation, Commissioning and Operation of a Beamlet Monitor in the negative ion beam test stand at NIFS, these proceedings paper O4-03

8. G. Serianni et al., Rev. Sci. Instrum. 85, 02 A736 (2014)

9. M. De Muri et al., IEEE Trans. Plasma Sci. 42, 1032-1035 (2014)

10. M. Brombin et al., IEEE Trans. Plasma Sci. 40, 724-729 (2012)

11. MDSplus official website: http://www.mdsplus.org

12. G. Serianni et al., AIP Conf. Proc. 1515, 579-586 (2013)

13. EXELIS Visual Information Solutions official website, www.exelisvis.com

14. P. R. Bevington, Data Reduction and error analysis for the physical sciences (McGraw Hill Book Company,1969)

15. S. Cristofaro, Characterisation of the BATMAN beam properties by Ha-Doppler shift spectroscopy and miniSTRIKE calorimeter. University of Padua, Master Thesis (2014)

16. F. Bonomo, BATMAN Beam Properties Characterization By The Beam Emission Spectroscopy Diagnostic, these proceedings paper P1-26 